\title{
LAS RELACIONES RUSO-ESPAก̃OLAS EN EL SIGLO XXI: UNA MIRADA DESDE RUSIA
}

\author{
Relations betueen Russia and Spain in the XXI \\ Century: A Vieu from Russia
}

\author{
Georgy Filatov \\ Instituto de la historia universal de la Academia de Ciencias de Rusia \\ E-mail: georgefilatov@gmail.com
}

\begin{abstract}
A principios del siglo XXI después de superar la crisis económica rusa de los 90 se estrecharon los lazos entre Rusia y España: aumentó el volumen de negocios y el flujo de turistas rusos al país ibérico. Los rusos empezaron a invertir activamente en el mercado inmobiliario español. En el ámbito de la política internacional, los Estados coincidían en la lucha contra el terrorismo internacional y la crítica del separatismo. Sin embargo, tenían serias discrepancias. Las autoridades rusas se mostraron en contra de la participación activa de España en la invasión estadounidense de Irak en 2003. Pero la etapa más difícil en las relaciones empezó después de la crisis ucraniana de 2014, cuando los países occidentales, incluida España, impusieron sanciones a Rusia. Sin embargo, a pesar de las tensiones entre Rusia y la Unión Europea, Madrid mantiene una relación especial con Moscú. El Gobierno español acogió submarinos rusos en Ceuta.
\end{abstract}

At the beginning of the XXI century the ties between Russia and Spain were tightened after overcoming the Russian economic crisis of the 1990s: trade and the flow of Russian tourists to the Iberian country increased. The Russians began to actively invest in the Spanish real estate market. In the field of international politics, States agreed on the fight against international terrorism and the critique of separatism. However, they had serious discrepancies. The Russian authorities were against the active participation of Spain in the US invasion of Iraq in 2003. Nevertheless, the most difficult stage in relations began after the Ukrainian crisis of 2014, when Western countries, including Spain, imposed sanctions on Russia. However, despite the tensions between Russia and the European Union, Madrid maintains a special relationship with Moscow. The Spanish government hosted Russian submarines in Ceuta.

España; Rusia; relaciones bilaterales; turismo.

Spain; Russia; bilateral relations; turism.

Key words 


\section{Introducción}

Las relaciones ruso-españolas en el siglo XXI han pasado tanto por momentos de gloria como de retroceso y tensión. Han estado influenciadas por el desplome del sistema bipolar y la posición versátil de Rusia en el escenario internacional en las primeras décadas del siglo XXI. En este periodo la economía rusa salió de la crisis de los años 90 lo que permitió consolidar los vínculos entre los dos países. Se pueden destacar dos periodos en las relaciones ruso-españolas en el siglo XXI: el primero, entre los años 2000 y 2014 y el segundo, desde el año 2014 hasta el momento presente.

El primer periodo se vio marcado por la potenciación y el desarrollo de las relaciones económicas y políticas entre las dos naciones, mientras que el segundo se caracterizó por una ralentización de dichos vínculos interestatales como consecuencia de la crisis general en las relaciones entre Rusia y el Occidente, causada por la situación en Ucrania y Crimea.

Los países de la Unión Europea impusieron sanciones a Rusia, mientras que Moscú respondió con el embargo a varios productos procedentes de las naciones que tomaron esa decisión. España, como miembro de la UE, ha seguido la política de este bloque respecto a Rusia. No obstante, la postura de Madrid ha sido bastante diferente a la de los "pesos pesados" de Europa como es el caso de Francia, Alemania y el Reino Unido. España, al igual que otros países del sur de Europa como, por ejemplo, Italia y Grecia, ha visto sin mucho entusiasmo las sanciones contra Rusia y, en la medida de lo posible, resistió a su implementación. En el caso de Grecia e Italia tal postura parece bastante lógica ya que estos países cuentan con una larga historia de relaciones con Rusia. Tanto Roma, como Atenas desde hace décadas, suministraban a Rusia productos de maquinaria y agricultura. Por su parte, Rusia es un importante suministrador de recursos energéticos para estos países, al igual que lo ha sido la Unión Soviética. Mientras tanto, la actitud benevolente de Madrid no es tan fácil de explicar. El volumen del intercambio comercial no representa un valor importante para las economías de los dos Estados. Las posturas políticas, asimismo, son muy diferentes. Es por eso que surge la pregunta sobre los motivos de una actitud tan especial de España hacia Rusia. Para explicar este fenómeno habría que prestar atención a los aspectos de las relaciones bilaterales que representan el mayor interés para ambos países. En el caso de España se trata principalmente del turismo y la actividad de los ciudadanos rusos en el mercado inmobiliario del país ibérico. Otro punto interesante es que Madrid aprovecha las relaciones con Moscú como una herramienta de presión sobre sus socios de la OTAN y la UE.

\section{Prehistoria de las relaciones}

Las relaciones entre Rusia y España siempre representaron cierta dificultad para dichos Estados, ubicados en dos extremos opuestos del continente europeo. La situación geográfica no ayudaba al progreso de los vínculos bilaterales en los tiempos en los que el transporte no estaba lo suficientemente desarrollado. Los avances tecnológicos del siglo XX acortaron las distancias haciendo posible un mayor acercamiento entre Moscú y Madrid y, sin embargo, este periodo histórico resultó todavía más complicado para las relaciones interestatales. La Revolución Rusa y la llegada al poder de los bolcheviques dejaron al país en un aislamiento internacional que tardó mucho tiempo en ser superado. España estableció las relaciones con la URSS solo en los años de la Guerra Civil, de 1936-1939, cuando se dio un repunte de los contactos bilaterales.
Se pueden destacar dos periodos en las relaciones rusoespañolas en el siglo XXI: el primero, entre los años 2000 y 2014 y el segundo, desde el año 2014 hasta el momento presente 
Moscú prestaba ayuda a la Segunda República con armamento y especialistas pero la victoria de los franquistas volvió a distanciar los dos países por un largo periodo de tiempo.

Los años de "tardofranquismo" es cuando empiezan a sentarse las bases de las relaciones entre Moscú y Madrid. Precisamente entonces tanto la política como la ideología pasaron al segundo plano, mientras que se priorizaron las perspectivas de la colaboración en el ámbito económico y, de no menos importancia, en el cultural. En la segunda mitad de la década de 1960, la URSS y la España franquista negociaron convenios marítimos que permitieron el despliegue de la flota pesquera soviética en las islas Canarias (Yányshev Nésterova, 2016) y al mismo tiempo los suministros de los productos petrolíferos llegaron a dominar las exportaciones soviéticas a España. Por su parte, Madrid exportaba al país soviético productos de la agricultura y ciertas mercancías de la industria ligera. (Statistica, 1975, p. 155). En 1972 fue firmado el Tratado Comercial y finalmente en 1977 se restablecieron las relaciones diplomáticas.

Hasta finales de los años 80, los vínculos entre ambos países se limitaban generalmente al sector económico y el cultural. Hasta la misma desintegración de la URSS los países intercambiaban delegaciones culturales. En el ámbito económico, Moscú seguía suministrando a España productos petrolíferos mientras que la última empezó a exportar, asimismo, mercancías de la industria pesada.

Por ejemplo, a mediados de los años 80 la Unión Soviética compró a España buques cisterna para el transporte de sustancias químicas (Dubinin, 1999, p. 317). Tampoco faltaron momentos complicados. Moscú no vio con buenos ojos que España entrara en la OTAN en 1982 mientras que en la prensa española de vez en cuando aparecían especulaciones sobre la implicación de la URSS en la actividad de la organización terrorista ETA que cobró la mayor fuerza precisamente en la primera mitad de los años 80 .

Al parecer, la desintegración de la URSS eliminaba el último obstáculo para el progreso de las relaciones ruso-españolas (el sistema político que contrarrestaba a todos los países capitalistas) pero la consiguiente crisis en la economía rusa que duró casi una década volvió a minimizar las relaciones entre los dos Estados. Las primeras mejoras empezaron a apreciarse a finales de la década de 1990 (Yákovlev, 2011) y ya a principios del XXI las relaciones ruso-españolas entraron en auge.

\section{Intensificación de las relaciones (2000 - 2014)}

Así como el estancamiento en las relaciones entre Moscú y Madrid se produjo principalmente como resultado de los problemas en la economía rusa, la recuperación de la última inevitablemente llevaba a la intensificación de los contactos. El crecimiento de los precios de petróleo, el producto clave de las exportaciones rusas, al igual que una serie de reformas realizadas en los primeros años del Gobierno de Vladímir Putin, permitieron estabilizar tanto el sistema económico como el jurídico del país (Sutela, 2014). Dichos avances tuvieron un efecto positivo sobre el desarrollo de los vínculos comerciales entre Rusia y España que se manifestó, sobre todo, en la dinámica positiva del volumen de intercambio comercial.

A partir del año 1999 este aumentó 7 veces alcanzando 7 mil millones de euros para el año 2014 después de estar la segunda mitad de los años 90 sin traspasar mil millones de euros (DataComex, Comercio exterior Español con Rusia, 2018). Sin embargo, esta cifra estaba muy por debajo del intercambio comercial de Rusia y sus principales socios de la UE, como por ejemplo, Alemania (60 mil millones de euros) e Italia (40 mil millones de euros) (Rosstat, 2018).
Hasta finales de los años 80, los vínculos entre ambos países se limitaban generalmente al sector económico y el cultural. 
La base de las exportaciones rusas a España la forman el petróleo y sus derivados al igual que los metales ferrosos y de color. España, por su parte, exporta a Rusia automóviles, máquinas industriales y productos alimenticios. Esa estructura de las relaciones comerciales entre los dos países se remonta aún a la época soviética y hasta el momento presente no ha experimentado cambios considerables. Un elemento nuevo en los vínculos bilaterales llegó a ser el turismo ruso en España. Asimismo, los turistas soviéticos visitaban el país ibérico, pero su cantidad fue muy insignificante, sobre todo para un país que desde los años 70 ha sido uno de los principales destinos turísticos del mundo.

Así, por ejemplo, fueron 5.500 soviéticos los que visitaron España en el año 1982 (AVP, Informe sobre relaciones bilaterales, 1983) de un total de 43 millones de personas (Shubert, 1990, p. 305) que viajaron al país en el mismo año, con lo cual los ciudadanos soviéticos formaron solo un $0,01 \%$ de la cantidad general de turistas. En los próximos años la situación no cambió considerablemente. Una cantidad tan insignificante de turistas soviéticos tiene que ver, ante todo, con las restricciones existentes en la URSS respecto a la salida de sus ciudadanos al extranjero. La crisis económica de principios de los años 90 y la del año 1998 tampoco ayudaban a la salida masiva de los rusos al extranjero. Pero en la segunda mitad de la primera década del siglo XXI, la situación cambió radicalmente. El flujo de los turistas rusos al país ibérico iba en aumento. Si en 2002 fueron 253.000 turistas rusos los que visitaron España, en 10 años esa cantidad sumó un millón de personas llegando a 1,2 millones de personas en el año 2012.

En el año 2013 se registró el máximo histórico de 1,5 millones de turistas rusos que viajaron a España. Sin embargo, hay que tener en cuenta que, por su cantidad, los rusos no podían compararse con los representantes de otros países como, por ejemplo, Alemania con 9,8 millones de turistas o el Reino Unido con 14,3 millones. Los rusos formaron solo 2,6\% de los turistas que recibió España en aquel año (60,7 millones) (FRONTUR, 2013). Sin embargo, los documentos elaborados por las instituciones públicas de España prestaban una atención especial a los turistas de Rusia. Así, por ejemplo, en el Plan Nacional e Integral de Turismo 2012-2015 preparado por el pertinente ministerio, los turistas rusos figuraban como el consumidor más prometedor de los servicios turísticos de España (Ministerio de Energía, Turismo y Agenda Digital, 2015, p. 30). Tal actitud se debía a un rápido crecimiento de la cantidad de turistas rusos en 2012-2013. En 2012 el aumento alcanzó 39\% y en 2013, 31\%.

Por otra parte, los informes del Ministerio de Turismo señalaban que durante su estancia en España los ciudadanos rusos gastaban más que el promedio de los turistas (Instituto de Estudios Turísticos, 2012, p. 7), a saber, aproximadamente 115 euros al día sin gastos de transporte, frente a 78-80 euros que solían gastar los visitantes de otros países. En total, los turistas rusos gastaron en España 2,2 mil millones de euros en 2012 (Sánchez Andrés \& Pedro, 2015, p. 2). De esta forma en aquel momento los rusos generaban mucha expectativa como clientes para los destinos turísticos de España.

Al mismo tiempo, los ciudadanos rusos empezaron a intervenir en el mercado inmobiliario del país ibérico. En 2013, estos ocuparon el tercer puesto en cuanto a la compra de inmuebles en España después de los ingleses y los franceses que tradicionalmente lideraban este sector. En total, según las estadísticas inmobiliarias, los ciudadanos rusos compraron en aquel año casi $1 \%$ de todos los inmuebles vendidos en el país (Colegio de Registradores de la Propiedad, Bienes Muebles y Mercantiles de España, 2014, p. 152). 
En el ámbito político, Moscú y Madrid coincidían en sus posturas acerca de varios asuntos de importancia. Por ejemplo, ambos países compartían la preocupación frente a la amenaza del terrorismo internacional. Tanto Rusia como España vivieron sangrientos atentados que dejaron una gran cantidad de víctimas. En Rusia ha sido el caso de la crisis de rehenes del teatro Dubrovka de Moscú, la masacre de la escuela de Beslán, al igual que una serie de explosiones que se cobraron las vidas de decenas de personas. En España, el principio de la década del 2000 se caracterizó por un aumento de la actividad de la organización terrorista vasca ETA. Entonces en los años 2000 y 2001 fallecieron 23 y 15 personas respectivamente (Fundación Víctimas del Terrorismo, 2018). En 2004, el país ibérico vivió los atentados terroristas del 11M.

Aparte del terrorismo, tanto Moscú como Madrid enfrentaron el problema del separatismo. Ambos países condenaban la posible aparición de precedentes secesionistas internacionales. Por ejemplo, tanto Moscú como Madrid se negaron a reconocer la independencia de Kosovo. Al mismo tiempo, los dos países contaban con importantes divergencias. Las autoridades rusas adoptaron una posición crítica respecto a la participación activa de España en la intervención estadounidense en Iraq en 2003. Moscú se pronunciaba en contra de esta iniciativa de Washington. Este problema en las relaciones desapareció después de que, en 2004, España retirara sus tropas de este país de Oriente Medio.

A lo largo de la década del 2000, los contactos bilaterales iban en aumento. En 2006 el presidente de Rusia Vladímir Putin viajó a España con una visita oficial y en 2009 el presidente Medvédev hizo lo mismo.

De esta manera, los primeros catorce años del siglo XXI llegaron a ser un periodo de florecimiento en las principales esferas de las relaciones ruso-españolas. El intercambio comercial iba creciendo hasta alcanzar cifras récord en la primera mitad de la segunda década del siglo. Aumentó la importancia de los turistas rusos para España. El sector turístico español llegó a percibir el mercado ruso como uno de los más prometedores para su país. Los ciudadanos de Rusia se convirtieron en importantes actores en el mercado inmobiliario de España lo que fue muy oportuno para el país ibérico que estaba viviendo una crisis en este sector.

Pese a ciertas discrepancias políticas, las relaciones entre Moscú y Madrid siguieron siendo positivas a lo largo de todo este periodo, algo que fue confirmado por las mutuas visitas de los líderes nacionales. Sin embargo, los acontecimientos de la segunda mitad de la década se convirtieron en una importante prueba para las relaciones bilaterales.

\section{Las relaciones ruso-españolas después de 2014}

Los contactos entre Moscú y Madrid en el periodo posterior al año 2014 siguieron el paradigma general de las relaciones entre Rusia y los países del Occidente. Madrid como miembro de la Unión Europea no podría ir en contra de la política llevada a cabo por Bruselas. No obstante, la postura de España hacia Rusia era mucho más benevolente que la de los "pesos pesados" de la política europea como, por ejemplo, Alemania, Francia y el Reino Unido.

Se suele considerar que los acontecimientos en Ucrania en el año 2014 llegaron a ser el punto crucial para las relaciones ruso-españolas (Charap \& Shapiro, 2015). No obstante, las relaciones de Rusia con Estados Unidos y la UE ya habían empezado a empeorar (Haukkala, 2015), y el 2014 fue el año en que las divergencias entraron en su fase más aguda. La crisis en las relaciones ruso-españolas se manifestó en la caída de los principales indicadores, mientras
Aparte del terrorismo, tanto

Moscú como

Madrid enfrentaron el problema del separatismo. Ambos países condenaban la posible aparición de precedentes secesionistas internacionales 
que el mayor daño lo sufrió el comercio con productos alimenticios. Los países occidentales introdujeron sanciones contra los sectores clave de la economía rusa y Moscú respondió con el embargo a las importaciones de una serie de productos de la agricultura, siendo España uno de los países más perjudicados, ya que aún desde los tiempos soviéticos Rusia era uno de los importantes mercados para la agricultura del país ibérico (Filatov, 2017).

Según estiman los expertos, el daño de las sanciones rusas a España alcanza 550 millones de euros anuales (Otamendi García-Jalón, 2015). No obstante, conviene destacar que la disminución de las exportaciones de los productos alimenticios de España a Rusia comenzó en 2013, tras la restricción de importaciones de productos cárnicos aprobada por Moscú. En aquel entonces la medida se justificaba con razones sanitarias ya que a finales de 2013 se detectaron casos de peste porcina africana en Letonia y Polonia.

Los acontecimientos en Europa del Este fueron solo una de las causas que llevaron a la ralentización de las relaciones ruso-españolas. Las sanciones económicas contra Moscú impuestas en verano de 2014 afectaron su economía, pero esta todavía sufrió más por la caída de los precios de productos petrolíferos que se produjo a finales de 2014 (Tuzova \& Qayum, 2016). La posterior devaluación del rublo respecto al euro y la caída de la capacidad adquisitiva de los ciudadanos rusos causaron un perjuicio mucho mayor a los lazos económicos de los dos países. Precisamente esta fue la razón de la drástica caída de la cantidad de los turistas rusos en España que se observó ya en el año 2015, cuando solo 955.000 rusos visitaron el país ibérico (INE, 2016). Al mismo tiempo se aprecia el descenso de la presencia rusa en el mercado inmobiliario español. La parte de los ciudadanos rusos se redujo hasta 0,5\% en 2015 (Colegio de Registradores de la Propiedad, Bienes Muebles y Mercantiles de España, 2016, p. 66).

En 2016 el volumen del intercambio comercial disminuyó hasta 3 mil millones de euros. Sin embargo, es de notar que la caída se produjo principalmente debido a la desvalorización de las exportaciones rusas ya que España sigue exportando a Rusia más o menos la misma cantidad de productos que antes de la crisis, a excepción del sector alimenticio. Asimismo, el volumen de las exportaciones rusas se mantiene en el mismo nivel (Rosstat, 2018). La caída del equivalente monetario de las exportaciones rusas se explica por la devaluación del petróleo y sus derivados que son el principal producto exportado por Rusia a España.

Al mismo tiempo, pese a la gravedad de esta crisis y las tensiones en las relaciones bilaterales entre Rusia y la Unión Europea, en el marco de la cual la España moderna desarrolla su política exterior, Madrid sigue conservando una actitud especial hacia Moscú. Y eso a pesar de que España tuvo una actitud negativa hacia la situación con Crimea proyectándola, en parte, a sus propios problemas internos con Cataluña y el País Vasco (Sánchez Andrés \& Pedro, 2015, p. 4).

España reaccionó sin mucho entusiasmo a las sanciones impuestas por la UE contra Rusia (Sánchez Andrés \& Pedro, 2015, p. 4) tratando de disminuir la tensión que surgió como resultado de la crisis y las medidas adoptadas por la UE. Por ejemplo, a principios de 2015 el ministro de Asuntos Exteriores de España, José Manuel García-Margallo, declaraba que no hacían falta nuevas sanciones ya que los Acuerdos de Minsk sobre el cese del fuego en el Este de Ucrania "se estaban cumpliendo" (Colás, 2015).

Las autoridades españolas también manifestaron una actitud similar respecto a la posible imposición de nuevas restricciones en relación con los combates en Alepo a finales de 2016. Entonces Madrid declaró que solo apoyaría sanciones contra Rusia "si estas contribuyeran al cese

\section{LOS \\ acontecimientos en Europa del Este fueron solo una de las causas que llevaron a la ralentización de las relaciones ruso- españolas}


'inmediato’ de hostilidades en Alepo” (La Vanguardia, 2016). Una fórmula tan poco realista básicamente disimulaba la renuencia de España a apoyar las nuevas sanciones contra Rusia.

Al mismo tiempo Rusia y España mantienen colaboración incluso en las esferas que, al parecer, deberían haber sido afectadas en primer lugar. A pesar de que la situación en las relaciones entre Rusia y el Occidente a menudo sea caracterizada como una nueva guerra fría (Legvoldm, 2016), los contactos en la esfera militar siguen jugando un importante papel en los lazos entre Moscú y Madrid. Por ejemplo, 24 buques de guerra rusos pasaron por el puerto de Ceuta para el reabastecimiento en los años 2014 y 2015 (González, 2016). En el año 2016 el Gobierno español permitía el reabastecimiento de los submarinos rusos que iban desde la planta en el mar Báltico, donde se fabricaban, hacia su base permanente en el mar Negro.

Esto generó descontento en Estados Unidos. El senador estadounidense Joe Pitts incluso propuso una enmienda especial al presupuesto militar del país para el año 2017. Conforme a esta, el Ministerio de Defensa de EE. UU. tendría que notificar al Congreso estadounidense si algún país miembro de la OTAN permitía a los buques de guerra rusos entrar en sus puertos (Merrill, 2016). Otro motivo para el descontento fue el hecho de que las naves rusas hacían la parada cerca de la base militar británica en Gibraltar. Sin embargo, el caso no fue más allá de amenazas y acusaciones de haber traicionado la OTAN.

Mientras tanto, en los medios españoles se barajó un posible trasfondo de esta postura de España. Y es que, primero, Ceuta y Melilla no estarían amparadas por la OTAN en el caso de una agresión militar (Público, 2016). Segundo, a pesar de que la crisis en torno a Gibraltar ya se había atenuado, en España todavía existe un descontento respecto al control británico sobre esta península.

En los años 60, Gibraltar ha sido uno de los temas que permitieron empezar la reconstrucción de las relaciones entre la URSS y la España franquista

Es curioso que, aún en los años 60, precisamente Gibraltar haya sido uno de los temas que permitieron empezar la reconstrucción de las relaciones entre la URSS y la España franquista. En 1969 Madrid favoreció que los barcos pesqueros de la URSS usaran los puertos españoles y no los de Gibraltar para el reabastecimiento y el mantenimiento técnico. Como resultado de las negociaciones, se firmaron acuerdos que permitían a la Unión Soviética tener una base de su flota pesquera en las islas Canarias (Informe sobre la misión del ministerio de la flota marítima en España, 1969, p. 49). No obstante, a pesar de una actitud tan benévola de España hacia la colaboración con Rusia, la política exterior de Madrid no puede contradecir abiertamente a la postura occidental. Por ejemplo, cuando Rusia envió a las costas de Siria una escuadra con portaaviones "Admiral Kuznetsov", España le negó el acceso a sus puertos (González, 2016).

Todo esto plantea la pregunta de cuál es la raíz de las relaciones especiales entre Rusia y España. Podemos destacar tres motivos principales al respecto.

Primero, las premisas históricas. España y Rusia están geográficamente alejadas entre sí. Por lo tanto, no tienen ni han tenido graves conflictos que hayan influenciado de alguna manera sus relaciones.

Segundo, las razones económicas. Rusia no ha incluido en las listas de productos sancionados el vino y el aceite de oliva que forman una parte importante de las exportaciones de España.

Tercero, aunque los turistas rusos no puedan competir con los visitantes del Reino Unido y Alemania en términos cuantitativos, suelen gastar más por persona que los ingleses y los alemanes. Aquí también se pueden mencionar las inversiones de los rusos en el mercado inmobiliario de 
España. A pesar de que en este momento, dicho aspecto atraviese por un periodo complicado, se puede presumir que Madrid está esperando su recuperación.

Por lo tanto, queda claro que pese al empeoramiento de las relaciones entre Rusia y los países de Occidente tras los acontecimientos del año 2014, Madrid y Moscú mantienen un trato respetuoso y cálido. La caída del intercambio comercial y otros indicadores económicos tuvo que ver principalmente con la crisis económica en la que se sumió Rusia en el periodo de 2014-2016.

La caída de los precios de petróleo y la devaluación del rublo restaron las posibilidades de los rusos en cuanto a los viajes y la adquisición de los bienes en el extranjero. Sin embargo, las instituciones españolas siguen considerando el mercado turístico ruso como prometedor. El carácter singular de las relaciones ruso-españolas se confirma también con la persistente colaboración en el ámbito militar. Los navíos rusos siguen usando los puertos españoles para el reabastecimiento a la hora de transitar de los mares nórdicos al mar Mediterráneo y el mar Negro.

\section{Conclusión}

Por lo tanto, queda claro que las relaciones entre Rusia y España han tenido una buena dinámica desde el comienzo del siglo XXI. Hasta el año 2014, la colaboración entre los dos países iba creciendo al igual que el intercambio comercial, principalmente a costa de las exportaciones del petróleo ruso. Iba en aumento la cantidad de turistas rusos que llegaban a España a descansar. Los ciudadanos de Rusia se convirtieron en importantes actores en el mercado inmobiliario español. Los contactos políticos, asimismo, se producían con regularidad. Las dos naciones compartían la preocupación por ciertos problemas, en primer lugar, el del terrorismo internacional, y trataban de solucionarlos conjuntamente. Asimismo, coincidían en una actitud negativa hacia los movimientos independentistas. Todo ello permitió crear un sólido fundamento que seguiría ayudando a los países a promover sus relaciones después del 2014.

En la segunda mitad de la segunda década del siglo, los indicadores de la colaboración ruso-española se vieron seriamente perjudicados por la crisis. El intercambio comercial se redujo, al igual que la cantidad de turistas rusos en España. A pesar de que una de las causas que lo provocaron fue un empeoramiento general de las relaciones entre los países occidentales y Rusia ocasionado por los acontecimientos en Ucrania en 2014, el componente político no fue decisivo para los contactos ruso-españoles. Un efecto mucho mayor tuvo la crisis causada por la caída de los precios de petróleo. La capacidad adquisitiva de los rusos disminuyó haciendo que tuvieran menos posibilidades para viajar y adquirir bienes inmuebles. A pesar de ello, España espera la recuperación del flujo turístico desde Rusia. Los turistas de Rusia representan un interés para el Gobierno español no por su cantidad sino por gastar considerablemente más que el promedio de los visitantes de otros países. La colaboración militar de España y Rusia ha sido otro rasgo emblemático de las relaciones especiales entre Madrid y Moscú.

Resumiendo lo dicho, se puede destacar que los buenos vínculos económicos junto con un trato amistoso han cimentado los lazos entre los dos países desde el comienzo del siglo XXI, generando mayores expectativas para el futuro, sobre todo, en las esferas que despiertan el mayor interés de España como son el turismo y las inversiones en el mercado inmobiliario español. 


\section{Bibliografía}

AVP (Archivo de la política exterior de Rusia). Informe sobre relaciones bilaterales (1983), [Informe] F 97. Ser. 56. Exp.4. Caja 28. P. 90

Charap, S., \& Shapiro, J. (2015). Consequences of a New Cold War. Survival, 57(2), 37-46. DOI: https://doi.org/10.1080/00396338.2015.1026058

Colás, X. (10 de marzo de 2015). Margallo rechaza más sanciones contra Rusia porque los acuerdos de Minsk se están cumpliendo. El Mundo. Recuperado de http://www.elmundo.es/ internacional/2015/03/10/54fec9bbe2704e053e8b457c.html

Colegio de Registradores de la Propiedad, Bienes Muebles y Mercantiles de España. (2013). Estadística registral inmobiliaria annario 2012. Recuperado de http://www.registradores. org/wp-content/estadisticas/propiedad/eri/ERI_Anuario_2013.pdf

Colegio de Registradores de la Propiedad, Bienes Muebles y Mercantiles de España. (2016). Estadística registral inmobiliaria annario 2015. Recuperado de http://www.registradores. org/wp-content/estadisticas/propiedad/eri/ERI_Anuario_2015.pdf

DataComex. Comercio exterior español con Rusia 1995-2016. http://datacomex.comercio.es/ CabeceraPersonalizada.aspx

Dubinin, Y. (1999). Embajador! Embajador!: apuntes de un embajador en España. Moscú: Rosspen.

La Vanguardia. (2016). España sólo apoyará sanciones contra Rusia si contribuyen al cese «inmediato» de hostilidades en Alepo. Recuperado de https://www.lavanguardia.com/ vida/20161017/411066285264/espana-solo-apoyara-sanciones-contra-rusia-si-contribuyen-al-cese-inmediato-de-hostilidades-en-alepo.html

Instituto de Estudios Turísticos. (2012). Balance del turismo. Recuperado de http://estadisticas. tourspain.es/es-ES/estadisticas/analisisturistico/balantur/anuales/Balance\%20del\%20 turismo\%20en\%20Espa\%C3\%B1a.\%20A\%C3\%B10\%202012.pdf

INE. (2016). Estadística de Movimientos Turísticos en Fronteras (FRONTUR) http://www.ine.es/ $\mathrm{daco} / \mathrm{daco} 42 /$ frontur/frontur1215.pdf

Filatov, G. (2017). Economic relations between the USSR and the francoist Spain in the 1960s. Iberoamerikanskie tetradi, 4(18), 20-26.

FRONTUR. (2013). Movimientos turísticos en fronteras. Recuperado de http://estadisticas. tourspain.es/WebPartInformes/paginas/rsvisor.aspx?ruta=\%2fFrontur\%2fEstructura\%2f Anual\%2fEntradas+de+turistas+seg\%u00fan+Pa\%u00eds+de+Residencia.+-+Ref.203 \&par $=1$ \&idioma $=\mathrm{es}-\mathrm{ES} \&$ anio $=2014$

Fundación Víctimas del Terrorismo. (2018). Asesinatos ETA desde 1968 a 2010. Recuperado de http://fundacionvt.org/victimas-de-eta/

González, M. (27 de marzo de 2016). Ceuta, 'base’ de la flota rusa en el Estrecho. El País. Recuperado de https://politica.elpais.com/politica/2016/03/26/actualidad/1459022294_947252.html

González, M. (26 de octubre de 2016). España cancela la escala de la flotilla rusa en Ceuta ante las críticas de la OTAN. El País. Recuperado de https://elpais.com/internacional/2016/10/25/actualidad/1477421097_408325.html 
Haukkala, H. (2015). From Cooperative to Contested Europe? The Conflict in Ukraine as a Culmination of a Long-Term. Crisis in EU-Russia Relations. Journal of European Studies, 23(1), 25-40.

Público. (26 de octubre de 2016). La OTAN abronca a España por permitir repostar en Ceuta a una flota de guerra rusa. Recuperado de http:/www.publico.es/internacional/otan-preocupada-escala-buques-espana.html

Legvold, R. (2016). Return to Cold War. Malden: Polity Press.

Merrill, J. (27 de mayo de 2016). Spain 'betraying Nato allies' by hosting Russian navy. The Times. Retrieved from https://www.thetimes.co.uk/article/spain-betraying-nato-alliesby-hosting-russian-navy-v 5 kpktct 9

Ministerio de Energía, Turismo y Agenda Digital. (2015). Plan Nacional e Integral de Turismo 2012-2015. Recuperado de http://www.minetad.gob.es/turismo/es-ES/PNIT/Documents/ Informe\%20Final\%20del\%20PNIT.pdf

Otamendi, J. (2015). Efectos del veto ruso en las exportaciones españolas. Boletín económico de ICE, Información Comercial Española, 3063, 81-100.

RGANI. (Archivo estatal de la política contemporánea). Informe sobre la misión del ministerio de la flota marítima en España. (1969) [Carta]. RGANI, Fondo 5. Ser. 61. Exp. 590. Moscú.

Rosstat. (2018). El comercio exterior de la Federación de Rusia con los países extranjeros. Recuperado de http:/www.gks.ru/wps/wcm/connect/rosstat_main/rosstat/ru/statistics/ ftrade/\#

Sánchez Andrés, A., \& Pedro, N. de (2015). Spain and the European Union-Russia conflict: The impact of the sanctions. Notes internacionals CIDOB, 108 https:/www.cidob.org/ en/publications/publication_series/notes_internacionals/n1_108/spain_and_the_european_union_russia_conflict_the_impact_of_the_sanctions

Shubet, A. (1990). Historia social de España (1800-1990). Madrid: Nerea.

Statistica. (1976). Comercio exterior de la URSS en el año 1975. Moscú.

Sutela, P. (2014). The Political Economy of Putin's Russia. London: Routledge.

Tuzova Y., \& Qayum, F. (2016). Global oil glut and sanctions: The impact on Putin's Russia. Energy Policy, 90, 140-151. DOI: https://doi.org/10.1016/j.enpol.2015.12.008

Yákovlev, P. (2011). Rusia y España: de la asociación estratégica al partenariado para la modernización. Iberoamérica, 2, 47-69.

Yányshev Nésterova, I. (2016). "Sovhispan”: una joint venture hispano-soviética. Anuario de Estudios Atlánticos, 62, 1-22. 